exercises. Our key objectives were to 1) facilitate engagement of stakeholders and modelling teams, 2) develop a shared understanding of the current implementations of the NHSHCP, 3) identify what is working well and less well, 4) identify future hopes for the NHSHCP, and 5) explore features and specifications to potentially include in a useful decision-support tool for stakeholders.

Results Fifteen key stakeholders participated in workshop 1. They spanned all levels: local (NHS commissioners, GPs, academics), third sector organisations and national organisations (including PHE and NICE). This diverse mix of stakeholders provided a rich diversity of perspectives. Stakeholders agreed that there is continued (financial and political) support for the NHSHCP. However, many stakeholders highlighted issues with the lack of data on processes and outcomes, variety in quality of delivery and suboptimal public engagement. Stakeholders' hopes for the programme included maximising coverage, uptake and appropriate referrals, and producing additional evidence on population health, equity and economic impacts. Stakeholders suggested several useful features including focusing on feasible NHSHCP implementations based on goodpractice template scenarios, analysis of broader prevention activities at local level, accessible local data, broader economic perspectives and fit-for-purpose outputs. These results then informed the objectives for the second and subsequent project workshops, which will include sharing and refining a prototype user interface, and exploring different scenarios to be evaluated.

Conclusion This project includes innovative approaches to engage with key stakeholders via Hovmand style workshops. These potentially offer an effective participatory method for involving stakeholders in the process of understanding a complex problem, and collaborating in the joint development of a decision-support tool focused on the needs of the final users.

\section{P32 REALISING THE POTENTIAL OF THE WIDER PUBLIC HEALTH WORKFORCE: CAUTIONARY TALES FROM THE EVALUATION OF PROGRAMMES TO EXTEND THE ROLE OF FRONTLINE STAFF}

EC Goyder*, L Blank, A Haywood, E Holding. ScHARR, University of Sheffield, Sheffield, UK

\subsection{6/jech-2018-SSMabstracts. 158}

Rationale After the move of responsibility for public health to local government in 2013, Public Health England estimated there were 15 million people in the wider workforce who could potentially contribute to health improvement. In order to inform future initiatives to develop and extend the public health role of the wider workforce, we analysed data from the evaluation of two programmes designed to enhance the health promotion role of council employees, from a range of occupational groups. We explored the issues that need to be addressed to ensure this potential public health capacity can be appropriately harnessed.

Methods Qualitative data was collected from a) 12 staff focus groups undertaken during evaluation of 'Making Every Contact Count' (MECC) training offered to council staff b) indepth interviews with 21 neighbourhood housing officers undertaken for the evaluation of a new 'Housing+' service, which required them to provide holistic health and wellbeing advice to council housing tenants. The data was analysed thematically to identify potential barriers and facilitators to the engagement and development of the wider public health workforce.

Results The sample included staff from ten occupational groups with a wide range of roles and experience. Whilst most staff were positive in principle about engaging with health and wellbeing issues, occupational groups varied in their attitudes to the appropriateness of taking on a specifically health promoting role and a range of barriers and obstacles were identified. Obstacles included a lack of time and opportunity costs; conflict with other roles such as managing complaints and rent arrears; lack of capacity in other services they could refer clients to when urgent needs identified; and a concern they were being expected to replace other overstretched or non-existent community services. The range of views expressed on the feasibility and appropriateness of engaging clients or customers in discussion of health and wellbeing related issues, particularly health-related behaviour, suggested that implementation of training received, and the delivery of an extended role more generally, was likely to be highly variable.

Conclusion If the potential contribution of the wider workforce to maximising population health is to be achieved, the best way to engage staff and develop their role is likely to vary between occupational groups and the opportunity costs, potential unintended consequences and additional training needs must not be underestimated. Effectiveness and costeffectiveness cannot be assumed in the absence of evaluation of the wider impact of role development in terms of both intended and unintended consequences.

\section{P33 WHAT DO DOCTORS KNOW ABOUT THE CLINICAL COURSE OF RECOVERY FROM COMMUNITY-ACQUIRED PNEUMONIA?}

${ }^{1} \mathrm{C}$ Sabu Tharakan*, ${ }^{2}$ WS Lim, ${ }^{2} \mathrm{H}$ Pick, ${ }^{1} \mathrm{M}$ Bains, ${ }^{2} \mathrm{D}$ Ashton. ${ }^{1}$ School of Medicine, University of Nottingham, Nottingham, UK; ${ }^{2}$ Respiratory Medicine, Nottingham University Hospitals NHS Trust, Nottingham, UK

\subsection{6/jech-2018-SSMabstracts. 159}

Background Community-acquired pneumonia (CAP) poses a significant clinical and economic burden in the United Kingdom, with an annual incidence rate of $0.5 \%-1 \%$ in adults. The mortality rate upon hospitalisation ranges between 5\%$30 \%$. Return to baseline health following CAP can surpass the expected timelines of recovery, with respect to duration and symptom resolution. Little is known about doctors' knowledge in this area. Therefore, this study provides a previously unexplored qualitative insight into doctors' views and knowledge of the clinical course of recovery in patients with CAP, following discharge.

Methods Ten one-to-one semi-structured interviews were conducted with junior doctors, who had experience in managing patients with CAP. Interviews were recorded and transcribed verbatim. Data was analysed using thematic analysis, with a predominantly inductive approach.

Results Whilst doctors maintained consensus on their proficiency in diagnosis and treatment, their knowledge and awareness of clinical recommendations for post-discharge care were less comprehensive and definitive.

No patient information resources on the recovery process from CAP were mentioned, even though many believed this would be beneficial. Similarly, doctors highlighted a lack of 